\title{
Classical Trajectory Monte Carlo simulation of coincidence experiments in electron impact ionization of helium ${ }^{\star}$
}

\author{
Károly Tőkési ${ }^{1,2}$, Béla Paripás ${ }^{3, a}$, and Endre Kovács ${ }^{3}$ \\ 1 Institute for Nuclear Research, Hungarian Academy of Sciences (Atomki), 4026 Debrecen Bem tér 18/c, Hungary \\ 2 ELI-ALPS, ELI-HU Non-profit Ltd., Dugonics tér 13, 6720 Szeged, Hungary \\ 3 Institute of Physics, University of Miskolc, 3515 Miskolc-Egyetemváros, Hungary
}

Received 15 November 2018 / Received in final form 11 February 2019

Published online 17 April 2019

(c) The Author(s) 2019. This article is published with open access at Springerlink.com

\begin{abstract}
The state-to-state (exchange) interference of the autoionizing resonances of helium is studied in (e,2e) experiments. These studies are disturbed by the coincidence events caused by the direct ionization, so their decrease is desirable. For this reason, to mimic the experimental observation, we performed fourbody classical trajectory Monte Carlo calculations. The calculations were done for $93.15 \mathrm{eV}$ primary energy, where the exchange interference of the $2 \mathrm{~s}^{2}\left({ }^{1} \mathrm{~S}\right)$ and $2 \mathrm{p}^{2}\left({ }^{1} \mathrm{D}\right)$ autoionizing states of helium is expected. The yields of non-coincidence and coincidence events detected in various combinations of scattering geometry were calculated and compared with the experimental observations.
\end{abstract}

\section{Introduction}

Our intention with the coincidence electron energy spectra measured after electron impact excitation of helium is the study of state-to-state (exchange) interference. This type of interference can occur when two autoionizing resonances decay into the same final ionic state [1]. This interference can occur exclusively at a given (critical) electron impact energy when the electron pairs coming from the two reaction paths are indistinguishable. Namely, the energy of the scattered electron from one reaction path equals with the energy of the ejected electron released along the other path and vice versa. These electron pairs can be observed in coincidence by two electron spectrometers.

Along this line, we considered the possible state-to-state interferences of the $2 \mathrm{~s}^{2}\left({ }^{1} \mathrm{~S}\right)$ and $2 \mathrm{p}^{2}\left({ }^{1} \mathrm{D}\right)$ autoionizing resonances of He. These states decay to the same $\mathrm{He}^{+} 1 \mathrm{~s}^{-1}$ final state. According to the energy level diagram (see Fig. 1) the critical energy, where the state-to-state interference can occur is $93.15 \mathrm{eV}$. At this primary energy the energy of the scattered electron from the $2 \mathrm{~s}^{2}\left({ }^{1} \mathrm{~S}\right)$ reaction path $(33.25 \mathrm{eV})$ equals the energy of the ejected electron released along the other $2 \mathrm{p}^{2}\left({ }^{1} \mathrm{D}\right)$ path at $35.31 \mathrm{eV}$ and vice versa.

The ionization of helium can also happen directly at any primary energies above the ionization threshold $(24.59 \mathrm{eV})$. The excess energy can be shared between the two electrons at any rates. At $93.15 \mathrm{eV}$ primary energy the

* Contribution to the Topical Issue "Many Particle Spectroscopy of Atoms, Molecules, Clusters and Surfaces (2018)", edited by Károly Tőkési, Béla Paripás, Gábor Pszota, and Andrey V. Solov'yov.

a e-mail: fizpari@uni-miskolc.hu excess energy is $68.56 \mathrm{eV}$ which can be shared as $33.25 \mathrm{eV}$ and $35.31 \mathrm{eV}$, too. These two electrons are detected by the coincidence system, if they are emitted towards the entrances of the spectrometers. These coincidence events disturb the study of state-to-state interference, so their decrease is desirable. For this reason we have to optimize and find the angular domain pairs (from the realizable ones of our system) where these disturbing coincidence events are minimal. The expected coincidence data can be estimated theoretically by the integration of corresponding (6 fold) differential cross section (DCS) to the actual angular domains and energy ranges.

The theoretical treatment of the problem is extremely difficult because it requires accounting for the many body character of the collision system. This behavior is even more significant for light particle impact where the projectile trajectory cannot be approximated by a straight-line trajectory, as is done for heavy projectile impact. Therefore, the success of different approaches strongly depends on their ability to describe the many body character of the collision. The classical trajectory Monte Carlo (CTMC) method has been quite successful in dealing with both ionization and capture processes in ion atom collisions [2-4]. It was also shown that the CTMC method can be applied to light projectile impact as well [5-10]. It is a nonperturbative method. All interactions between the colliding partners can be taken into account exactly during the collision.

In this work the collision between electron and helium atom is studied classically. To mimic the experimental observation we performed four-body classical trajectory Monte Carlo calculations. The yields of non-coincidence and coincidence events detected in various combinations 


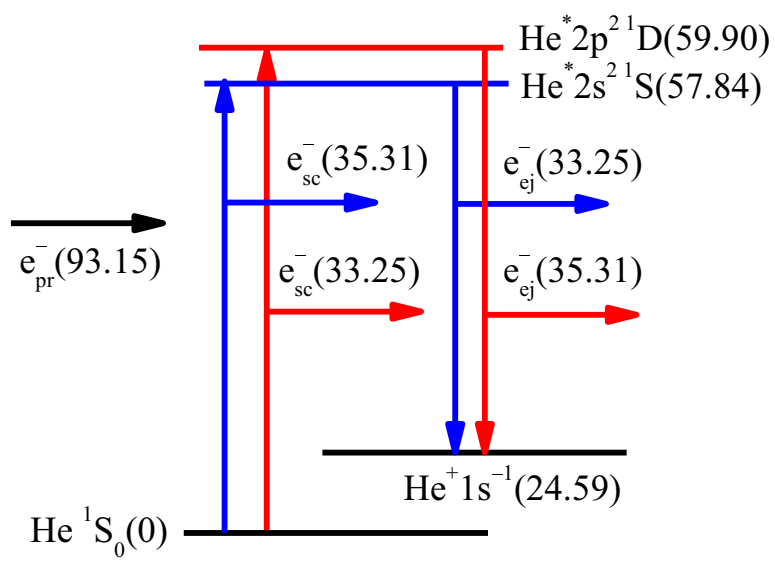

Fig. 1. The energy levels and transitions of the studied stateto-state interference.

of scattering geometry were calculated and compared with the experimental observations.

\section{Experimental}

Our experimental setup consists of two cylindrical mirror (CMA) spectrometers on a common axis, both contains two "box" type analyzers [11,12] in series (see Fig. 2). Spectrometer B (the right side) has a bit weaker energy resolution $(0.78 \%$ full widths at half maximum (FWHM) at $200 \mathrm{eV}$ ) than the A spectrometer (left side, $0.55 \%$ FWHM at $200 \mathrm{eV}$ ). The common axis of spectrometers is perpendicular both to the target gas beam and to the projectile electron beam, these three perpendicular lines cross each other exactly at the common focal point of the spectrometers.

The magnetic field inside the spectrometers and the interaction region is reduced to below $1 \mu \mathrm{T}$ by coaxial $\mu$-metal shielding layers and by adjusting the spectrometer axis into the optimal direction to the Earth magnetic field.

Large acceptance solid angles of CMA, essential to accumulate statistically significant data in reasonable time, are provided by $5^{\circ}$-wide entrance cones. The entrance angle with respect to the CMA axis is close to $\alpha_{0}=43.5^{\circ}$. When the entrance cone is open all round $\left(\phi=360^{\circ}\right)$, the acceptance solid angle is approx. $0.36 \mathrm{sr}$ (for the spectrometer A). In this case the scattering angle of the detected electrons (relative to the primary electron beam) is in the $47^{\circ}-133^{\circ}$ range. In the case of $B$ spectrometer the half of entrance cone (the forward and the backward quarters) has been closed, hereby the acceptance solid angle is reduced to $0.18 \mathrm{sr}$, and the scattering angle range to $65^{\circ}-115^{\circ}$.

The scattered or ejected electrons which enter the analyzers having the corresponding energy can pass the analyzers and they are detected by channel electron multipliers (CEM). The pulse counting of the amplified and formed CEM signals and the analyzer power supply control are performed by measuring cards controlled by a LabWindows code. The computer control involves simultaneous scan of both spectrometers through the desired energy ranges, even in opposite senses, if required. In the latter case the sum of the scattered electron energy and the ejected electron energy is kept constant (CIS (constant ionic state) coincidence (e,2e) measurements).

The $\vartheta$ scattering angle (the angle of the scattered or emitted electrons with respect to the primary beam) can be calculated from the angles $\alpha$ and $\phi$ :

$$
\cos \vartheta=\sin \alpha \cdot \cos \phi
$$

as it simply can be read from Figure 3. Based on this equation, the following scattering angular ranges can be obtained: in case of medium entrance angle $\left(\alpha_{0}=\right.$ $43.5^{\circ}$ ) for the forward sector $46.5^{\circ}-60.9^{\circ}$, for the upward and downward sectors $60.9^{\circ}-119.1^{\circ}$, and for the backward sectors $119.1^{\circ}-133.5^{\circ}$, respectively. In case we use the entire entrance angular range $\left(\alpha=41^{\circ}-46^{\circ}\right)$, then the scattering angular ranges expand as follows: forward sector $44^{\circ}-62.4^{\circ}$, the upward and downward sectors $59.4^{\circ}-120.6^{\circ}$, and the backward sectors $117.6^{\circ}-136^{\circ}$. Altogether, it can be said that the scattering angular ranges of the forward and backward sectors are much smaller $\left(\Delta \vartheta=14.4^{\circ}\left(18.4^{\circ}\right)\right)$ than the upward and downward sectors $\left(\Delta \vartheta=58.2^{\circ}\left(61.2^{\circ}\right)\right)$.

Figure 4 shows the typical electron spectra at different angular ranges, according to the sectors indicated in the bottom part in Figure 2 measured at $93.15 \mathrm{eV}$ primary energy. During these measurements, the current of the primary electron beam and its energy broadening were typically $50 \mathrm{nA}$ and $0.35 \mathrm{eV}$, respectively. Because of the sector dependent (and unknown) detection efficiency, the normalization of counts was essential. We performed it by the normalization of the sum of intensities of the four autoionizing (ejected) peaks. (Specifically the computer fitted $\mathrm{I} \mu$ intensity parameters were used [13]).

\section{Theory}

To mimic the experimental observation we performed four-body classical trajectory Monte Carlo calculations [14]. In our model the projectile is one particle (electron) and the target contains three particles, the helium core and two electrons. Figure 5 shows the relative position vectors of the four-body collision system. The binding energies of the target electrons to the target core are 0.903 a.u. and 2 a.u. according to the first and second ionization energy of helium. In the present case, all interactions among the particles are the pure Coulomb interactions except the interaction between the two target electrons. To avoid spontaneous autoionization this interaction is completely neglected during the calculations.

The initial conditions of the individual collisions are chosen at sufficiently large inter-nuclear separations such that the interaction between the projectile and the target is negligible. The initial states of the target are characterized by a micro-canonical ensemble constrained to the binding energies. In the present CTMC approach, Newton's classical nonrelativistic equations of motions for 


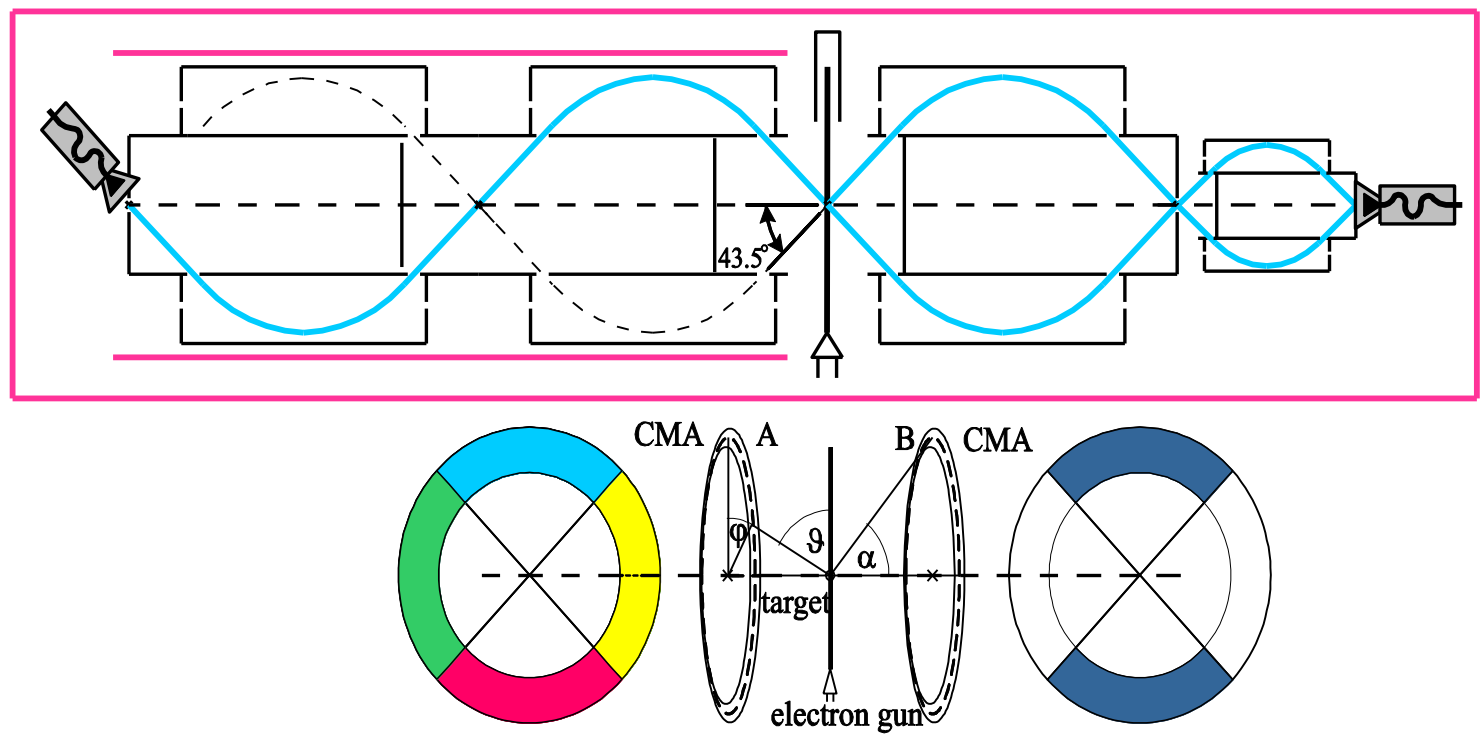

Fig. 2. Sketch of our (e,2e) coincidence spectrometer system.

a four-body system are solved [13]:

$$
m_{i} \frac{d^{2} \vec{r}_{i}}{d t^{2}}=\sum_{\substack{j=1 \\ j \neq i}}^{4} \alpha_{i j} Z_{i} Z_{j} \frac{\vec{r}_{i}-\vec{r}_{j}}{\left|\vec{r}_{i}-\vec{r}_{j}\right|^{3}} \quad(i, j=1, \ldots 4)
$$

where $m_{i}, \vec{r}_{i}$ and $Z_{i}$ denote the mass, position vector, and the charge of the ith particle, respectively. The $\alpha_{i j}$ are the switching parameters of the corresponding interactions defining the strength of the interaction among the particles. The value of $\alpha_{i j}$ is set equal to 1 when the interaction between the $i$ th and $j$ th particles is on and equal 0 when the interaction is off. Thus in our case $\alpha\left(e_{1}^{-}, e_{2}^{-}\right)=0$ ensures that we switch off the interaction between the two target electrons and mimic the independent electron approach in the helium atom. Then, Newton's classical non-relativistic equations of motions for a four-body system are solved numerically for a statistically large number of trajectories for given initial conditions until the converged final states are reached. Large numbers of classical trajectories were computed to calculate the total cross sections for the corresponding final channels. Large numbers of trials were required because the total cross sections are composed for many partial levels. Then the total ionization cross section was computed with the following formulas:

$$
\sigma=\frac{2 \pi b_{\max }}{T_{N}} \sum_{j} b_{j}^{(i)} .
$$

The statistical uncertainty of the cross section is given by

$$
\Delta \sigma=\sigma\left(\frac{T_{N}-T_{N}^{(i)}}{T_{N} T_{N}^{(i)}}\right)^{1 / 2} .
$$

In equations (2) and (3) $T_{N}$ is the total number of trajectories calculated for impact parameters less than $b_{\max }$, $T_{N}^{(i)}$ is the number of trajectories that satisfy the criteria for ionization, and $b_{j}^{(i)}$ is the actual impact parameter

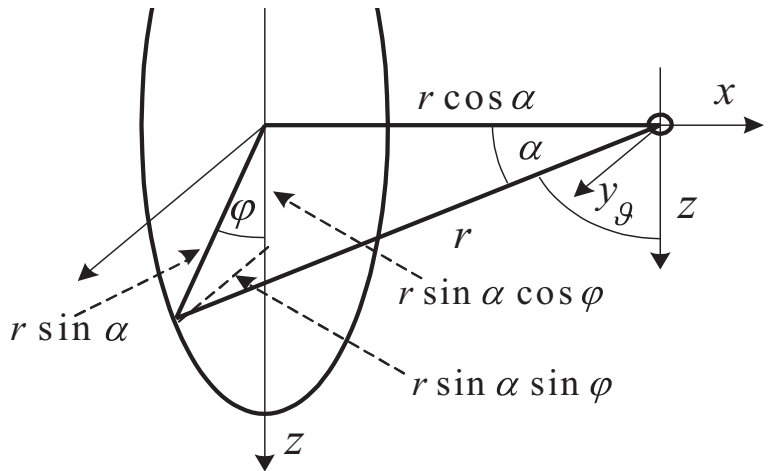

Fig. 3. Schematic diagram of the geometry of the scattering volume.

for the trajectory corresponding to the ionization process under consideration in the energy interval $\Delta E$ and the emission angle interval $\Delta \Omega$ of the electron.

\section{Results and discussion}

To study the collision between electron and helium atoms we have performed a classical simulation with an ensemble of $2 \times 10^{7}$ primary trajectories at $93.15 \mathrm{eV}$ energy. The calculations are based on the four-body model. Although we distinguish 9 different classical exit channels during the calculations in this work we focus on the net target single ionization channel:

$$
e^{-}+\mathrm{He}\left(1 s^{2}\right) \rightarrow \mathrm{He}^{+}+2 e^{-} .
$$

In this case, as a result of the collision between electron and helium atom, asymptotically we detect a single charged helium ion and two electrons. Classically, however this channel is a sum of two channels. We can call the first one as the one electron target loss channel. This channel 


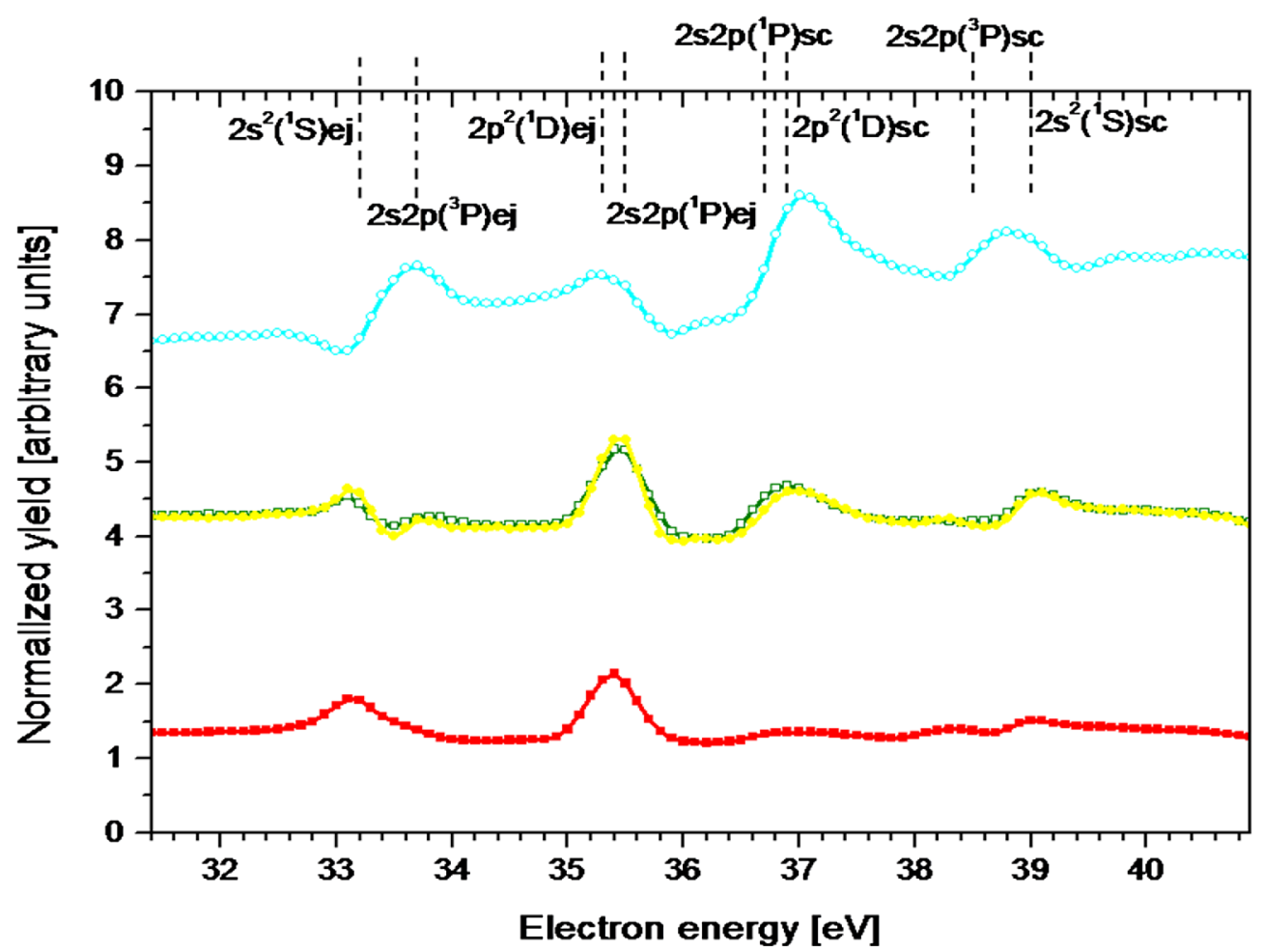

Fig. 4. The measured spectra at $93.15 \mathrm{eV}$ primary energy in the different angular ranges: forward (empty blue circle), upward (empty green square), backward (full red square), downward (full yellow circle). The yields are normalized by the sum of intensities of the four autoionizing (ejected) peaks. The position of the scattered (sc) and ejected (ej) electron peaks are shown by dashed lines.

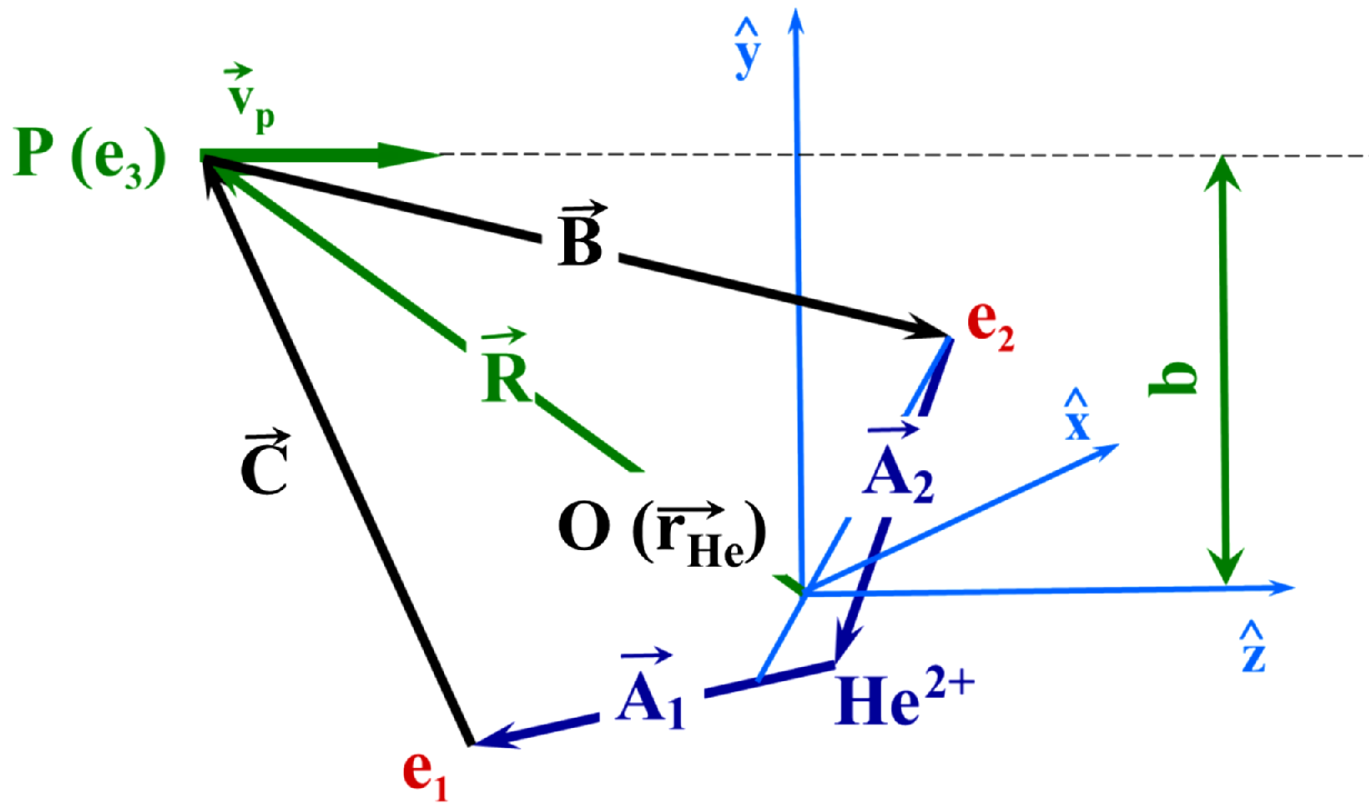

Fig. 5. The relative position vectors of the particles involved in 4-body collisions. $\vec{r}_{\mathrm{He}}$ is the position vector of the centre-of-mass of the target system, $b$ is the impact parameter. 
Table 1. The angular features of the sectors and their contribution to the total yield in different coincidence conditions.

\begin{tabular}{|c|c|c|c|c|c|c|}
\hline \multirow[t]{3}{*}{ Sector } & \multirow[t]{3}{*}{$\phi$} & \multirow[t]{3}{*}{$\vartheta$} & \multicolumn{3}{|c|}{ Calculated yield contribution at $33.24 \mathrm{eV}$} & \multirow{3}{*}{$\begin{array}{c}\text { Exp. non-coincident } \\
\text { ratio (from Fig. } 4 \text { ) } \\
\text { err. } \pm 2 \%\end{array}$} \\
\hline & & & \multicolumn{2}{|c|}{ Coincident } & \multirow{2}{*}{$\begin{array}{c}\text { Non- } \\
\text { coincident }\end{array}$} & \\
\hline & & & $\begin{array}{l}\text { Ionized electron } \\
\text { in A spectr. }\end{array}$ & $\begin{array}{l}\text { Projectile electron } \\
\text { in A spectr. }\end{array}$ & & \\
\hline Forward & $315^{\circ}-45^{\circ}$ & $46.5^{\circ}-60.9^{\circ}$ & $63.2 \%$ & $70.5 \%$ & $44.8 \%$ & $45 \%$ \\
\hline Upward & $45^{\circ}-135^{\circ}$ & $60.9^{\circ}-119.1^{\circ}$ & $14.7 \%$ & $12.6 \%$ & $22.3 \%$ & $23.5 \%$ \\
\hline Downward & $225^{\circ}-315^{\circ}$ & $60.9^{\circ}-119.1^{\circ}$ & $14.7 \%$ & $12.6 \%$ & $22.3 \%$ & $23.5 \%$ \\
\hline Backward & $135^{\circ}-225^{\circ}$ & $119.1^{\circ}-133.5^{\circ}$ & $7.4 \%$ & $4.3 \%$ & $10.6 \%$ & $8 \%$ \\
\hline
\end{tabular}

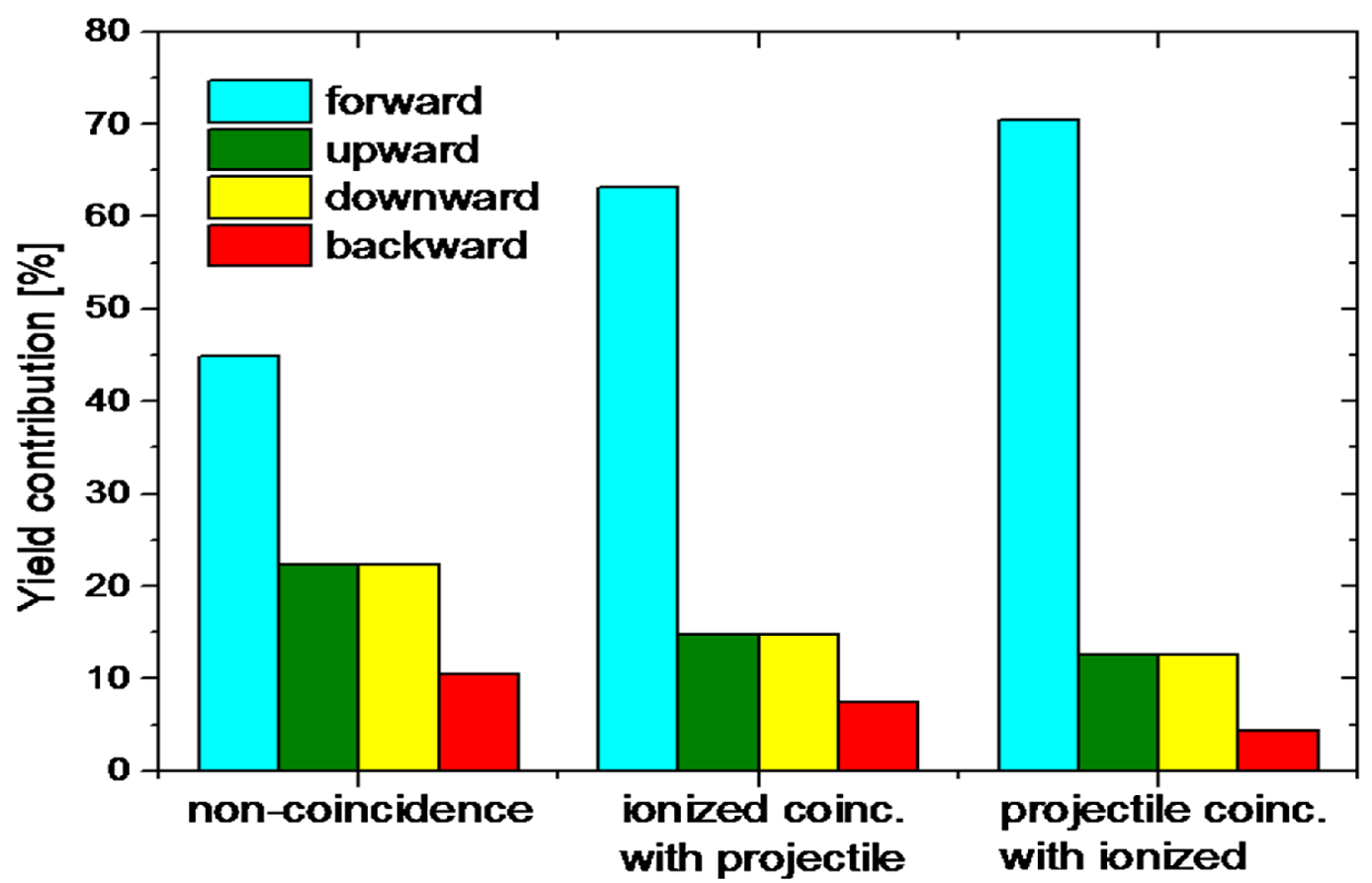

Fig. 6. The contribution of the sectors to the total yield in different coincidence conditions.

originates from a one step process. Due to the fact that classically the particle motions are deterministic and the electrons in the helium atom are distinguishable, we can define this channel as:

$$
e_{3}^{-}+\operatorname{He}\left(e_{1}^{-}, e_{2}^{-}\right) \rightarrow \operatorname{He}^{+}\left(e_{1}^{-}\right)+e_{3}^{-}+e_{2}^{-},
$$

and by

$$
e_{3}^{-}+\mathrm{He}\left(e_{1}^{-}, e_{2}^{-}\right) \rightarrow \mathrm{He}^{+}\left(e_{2}^{-}\right)+e_{3}^{-}+e_{1}^{-} .
$$

The second possible classical channel producing the same final particles as defined by equation (5) originates from the multi-electron interaction in a two-step process. We refer to it as target double ionization and at the same time projectile electron capture to the bound state of the target. We can define this channel as:

$$
e_{3}^{-}+\mathrm{He}\left(e_{1}^{-}, e_{2}^{-}\right) \rightarrow \mathrm{He}^{+}\left(e_{3}^{-}\right)+e_{1}^{-}+e_{2}^{-} .
$$

Performing the Monte Carlo simulation of 20 million collisions, it resulted 1.117 million net single target ionization events. From the outgoing $2 \times 1.117$ million electrons, with realistic angular and energy spread data, only 660 electrons (366 ionizing +294 scattered) are detected at $33.24 \mathrm{eV}$ by the four sectors of spectrometer A. On the other hand, no coincidence event is detected (none of their $35.32 \mathrm{eV}$ pairs is detected by spectrometer B). For this reason, in our coincidence calculations we used $5 \times$ entrance cone as for the angular window and $20 \times$ energy spread as the energy window.

With these larger conditions, we calculated the yields of non-coincidence and coincidence events detected in various combinations of sectors showing in Figure 2. The evaluation results can be seen in Table 1 and in Figure 6 . The four rows of the table concern the sectors of spectrometer $\mathrm{A}$, the coincidence condition is the detection in the upward and downward sectors of spectrometer B in every cases. (The emission angles for the upward and the downward sectors are the same, so their yields are not different). During the calculations the ionized and (scattered) projectile electrons are distinguished even when they have the same energy. For this reason the counts when the (different sectors of) spectrometer A detects ionized or projectile electrons are registered separately (columns fourth and fifth of Tab. 1). 
In the experiment, however, these two types are undistinguishable, therefore the average of these two percentages can compare with the experiments. In the last two columns the calculated and measured yields for the non-coincident case are compared. From these results we can conclude that our classical simulations for the non-coincidence case justify the experimental observations in Figure 4., i.e. the ratios of the continuous backgrounds (which come mainly from the direct ionization) are very close to the calculated non-coincident yields. It is not obvious, since the different autoionizing peaks do not have isotropic distribution. The angular distribution of their sum, however, is much smoother (at least in the measured angular range) than the distribution of ionization continuum.

Regarding the forward-backward asymmetry in coincidence experiments: on the basis of our calculations it is more significant than in non-coincidence experiments. In the given coincidence condition (the upward and downward sectors of spectrometer B) the ratio of the yields of the forward- and backward sectors is greater than 10. In our classical calculations the projectile- and the ionized electrons are distinguished, but during the measurements they are indistinguishable, so the average of the two coincidence yield is the proper value.

\section{Conclusions}

According to our CTMC calculations, the forwardbackward asymmetry of the electrons coming from direct ionization is greater in the coincidence experiments with the applied geometry than in non-coincidence experiments. Consequently, the direct ionization background in the forward sector of spectrometer A is very high, therefore this sector is less suitable for our state-to-state interference experiments. Although the background is minimal in the backward sector, the peaks of electrons scattered during the indirect (autoionizing) process are also low, which restricts the measure of possible state-to-state interference. The upward and downward sectors of spectrometer A (which are preferred in our coincidence experiments) mean really the best compromise, at least when the upward-downward sectors of spectrometer B are open.

Open access funding provided by University of Miskolc (ME). This research was supported by the European Union and the
Hungarian State, co-financed by the European Regional Development Fund in the framework of the GINOP-2.3.4-15-201600004 Project, aimed to promote the cooperation between higher education and industry. One of us (KT) was supported by the National Research, Development and Innovation Office (NKFIH) Grant KH 126886., by the COST Action CM1405 (MOLIM).

Open Access This is an open access article distributed under the terms of the Creative Commons Attribution License (http://creativecommons.org/licenses/by/4.0/), which permits unrestricted use, distribution, and reproduction in any medium, provided the original work is properly cited.

\section{Author contribution statement}

All authors discussed the results and contributed to the final manuscript. Károly Tokési performed the CTMC calculations.

\section{References}

1. J.P.V. den Brink, G. Nienhuis, J. van Eck, H.M. Heideman, J. Phys. B 22, 3501 (1989)

2. R. Abrines, I.C. Percival, Proc. Phys. Soc. 88, 861 (1966)

3. R.E. Olson, A. Salop, Phys. Rev. A 16, 531 (1977)

4. K. Tökési, G. Hock, Nucl. Instrum. Methods Phys. Res. B 86, 201 (1994)

5. D.R. Schultz, R.E. Olson, Phys. Rev. A 38, 1866 (1988)

6. K. Tőkési, Á. Kövér, J. Phys. B 33, 3067 (2000)

7. D.R. Schultz, R.E. Olson, C.O. Reinhold, J. Phys. B 24, 521 (1991)

8. R.A. Sparrow, R.E. Olson, J. Phys. B 27, 2647 (1994)

9. K. Tőkési, Á. Kövér, Nucl. Instrum. Methods Phys. Res. B 154, 259 (1999)

10. K. Tőkési, R.D. DuBois, T. Mukoyama, Eur. Phys. J. D 68, 255 (2014)

11. B. Paripás, B. Palásthy, G. Pszota, Eur. Phys. J. D 69, 34 (2014)

12. B. Paripás, B. Palásthy, M. Béres, Nucl. Instrum. Methods Phys. Res. B 369, 34 (2016)

13. B. Paripás, J.J. Jureta, B. Palásthy, B.P. Marinković, G. Pszota, J. Elect. Spectr. Relat. Phenom. 225, 10 (2018)

14. K. Tőkési, G. Hock, J. Phys. B 29, 119 (1996) 